\title{
Vasculogenic mimicry correlates to presenting symptoms and mortality in uveal melanoma
}

\author{
Shiva Sabazade ${ }^{1,2} \cdot$ Viktor Gill $^{2,3} \cdot$ Christina Herrspiegel $^{1,2} \cdot$ Gustav Stålhammar $^{1,2}$ (1)
}

Received: 13 October 2021 / Accepted: 4 November 2021 / Published online: 13 November 2021

(c) The Author(s) 2021

\begin{abstract}
Purpose Fluid-conducting extracellular matrix patterns known as vasculogenic mimicry (VM) have been associated with poor prognosis in uveal melanoma and other cancers. We investigate the correlations between VM, presenting symptoms, mortality, and the area density of periodic acid-Schiff positive histological patterns (PAS density).

Methods Sixty-nine patients that underwent enucleation for uveal melanoma between 2000 and 2007 were included. Clinicopathological parameters presenting symptoms and outcomes were collected. Histological tumor sections were evaluated for VM and PAS density was quantified with digital image analysis.

Results Thirty-four patients (49\%) presented with blurred vision. 18 (26\%) with a shadow in the visual field, 7 (10\%) with photopsia and/or floaters, and 2 (3\%) with metamorphopsia. Nine patients (13\%) had no symptoms at all. Median follow-up was 16.7 years (SD 2.6). A shadow in the visual field, but no other symptom, was positively correlated with the presence of $\operatorname{VM}(\varphi 0.70, p<0.001)$ and greater PAS density $(p<0.001)$. In multivariate regression, retinal detachment $(\mathrm{RD})$, presence of $\mathrm{VM}$, and PAS density $\geq$ median were independent predictors of a shadow, but not tumor distance to the macula, tumor apical thickness, tumor diameter, or ciliary body engagement. The presence of VM was associated with significantly shorter cumulative disease-specific survival (Wilcoxon $p=0.04)$, but not PAS density $\geq$ median, presenting symptoms or $\operatorname{RD}(p>0.28)$. Conclusion Tumors from uveal melanoma patients that report a visual field shadow are likely to display VM and greater PAS density, likely explaining the previously reported association between this symptom and poor prognosis.
\end{abstract}

Keywords Uveal melanoma $\cdot$ Pathology $\cdot$ Oncology $\cdot$ Vasculogenic mimicry $\cdot$ PAS density $\cdot$ Prognosis

\section{Background}

Uveal melanoma is the most common malignant intraocular tumor in adults. At the time of diagnosis, about $2 \%$ of patients have detectable metastases (Garg et al. 2021). However, within 15 years, between one-quarter and almost half all patients will succumb to metastatic disease with similar survival after eye-conserving plaque brachytherapy and surgical enucleation of medium sized tumors (Kujala et al. 2003; COMS 2006; Stalhammar 2020). This has been

Gustav Stålhammar

gustav.stalhammar@ki.se

1 St. Erik Eye Hospital, Stockholm, Sweden

2 Department of Clinical Neuroscience, Karolinska Institutet, Eugeniavägen 12, 17164 Stockholm, Sweden

3 Department of Pathology, Västmanland Hospital Västerås, Västerås, Sweden attributed to early seeding of micrometastases from the eye to distant organs, primarily the liver (Singh 2001; Callejo et al. 2007; Uner et al. 2021). Once these seeded clusters of dormant tumor cells grow into larger, radiologically detectable, macrometastases, median patient survival is a year or less (Khoja et al. 2019; Rantala et al. 2019).

Two of the strongest prognostic factors for the risk of developing macrometastases are the presence of periodic acid-Schiff (PAS) positive extracellular matrix patterns forming fluid-conducting channels (vasculogenic mimicry, VM), and the presence of a dense network of microvessels (Folberg et al. 1992, 1996; Foss et al. 1996; Mäkitie et al. 1999; Folberg and Maniotis 2004; Cao et al. 2013; Stålhammar et al. 2019).

Presenting symptoms of uveal melanoma include blurred vision, photopsia, floaters, visual field defects, pain, and metamorphopsia (Damato and Damato 2012). In a recent report, we showed that a shadow in the visual field as a presenting symptom was associated with exudative retinal 
detachment (RD, Fig. 1) and larger tumor size, and that it was an independent predictor of uveal melanoma-related mortality, regardless of other simultaneous symptoms, tumor size, tumor location, local extent, and stage (Fili et al. (2021)). In univariate analysis, there has been conflicting reports on the prognostic significance of RD; some studies have found that it is a significant risk factor for tumor-related death, whereas others have indicated the contrary (Packer et al. 1992; Foss et al. 1997a, b). Regardless, there is little reason to believe that RD is directly linked to prognosis. Rather, it may be a marker for other tumor characteristics that are in turn more closely related to the risk for metastasis and tumor-related death. As indicated by multivariate regression analyses, the correlation may be related to tumor size and the presence of microvascular loops and networks (Kivela et al. 2001).
As a shadow in the visual field at presentation of uveal melanoma has been shown to be associated with retinal detachment as well as poor prognosis, and retinal detachment with tumor size and vascularity, then the shadow in the visual field should also be associated with tumor vascularity. In this paper, we therefore examine this correlation. Patients' reports of their symptoms at the point of enucleation of an eye with uveal melanoma are correlated to histological findings including the presence of VM and the density of PASpositive patterns.
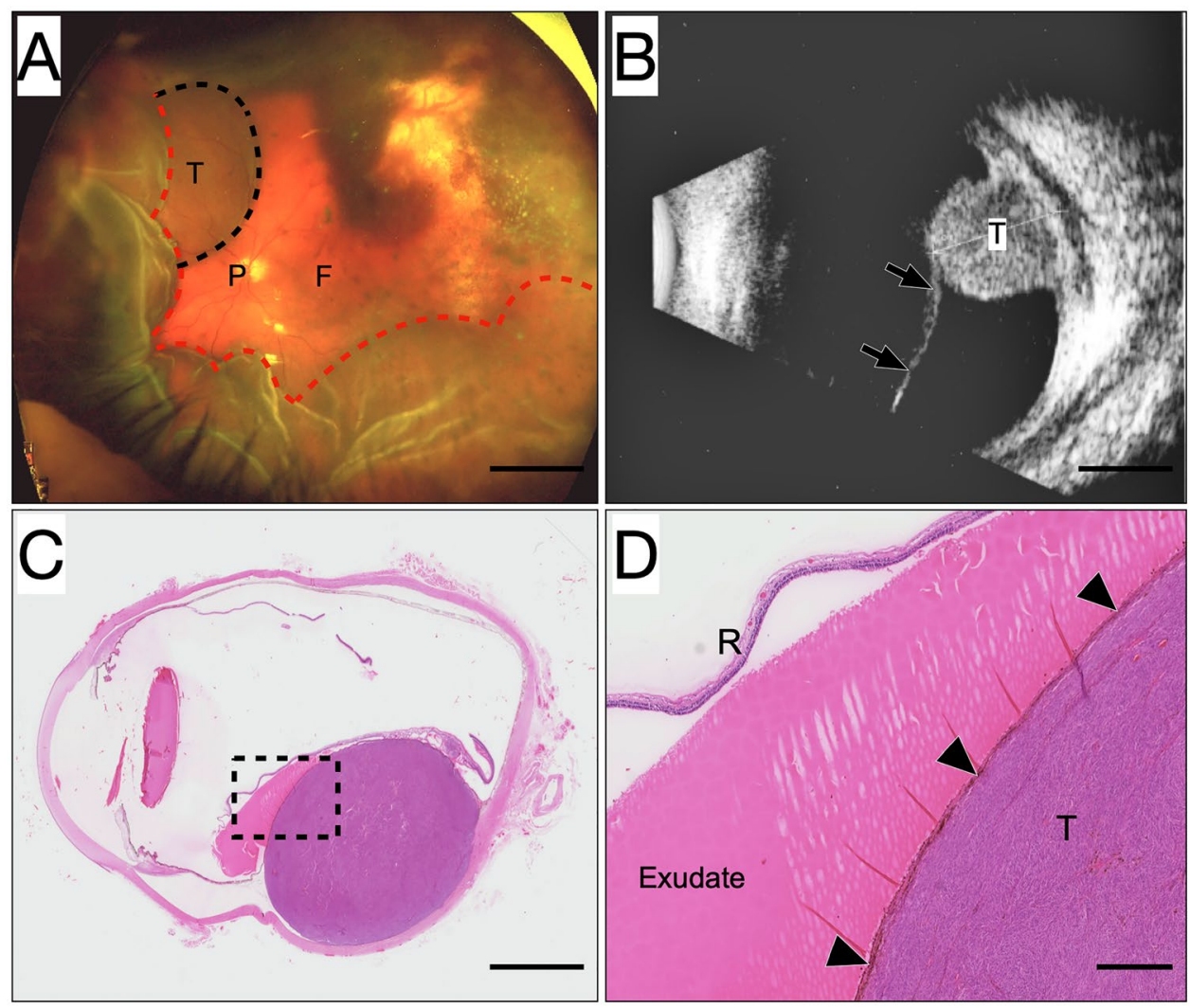

Fig. 1 Examples of tumor-associated exudative retinal detachment. A Appearance in wide-field fundus photography (Optos, Inc, Dunfermline, UK). A pigmented uveal melanoma ( $\mathrm{T}$, outlined by the black dotted line) is situated superionasally to the optic disc (P) and fovea $(F)$. Partially pigmented inactive tumor remnants surrounding a yellow choroidal atrophy associated with previous plaque brachytherapy are seen superiotemporally, indicating that the present tumor was a relapse outside of the treated area. Much of the nasal, temporal, and inferior retina have detached in an exudative retinal detachment by fluid leaking from the tumor (outlined by the red dotted line). B In ultrasonography, the detached retina (arrows) can be seen extending inferiorly from the tumor (T) apex. C Histological appearance. Tissue section stained with hematoxylin and eosin and digitally scanned at $\times 400$ (Nano Zoomer 2.0 HT, Hamamatsu Photonics K.K., Hamamatsu, Japan). A large dome-shaped melanoma is located in the posterior aspect of the choroid. The normal shape of the sclera has been artefactually deformed. Scale bar $5 \mathrm{~mm}$. D Magnification of the area within the dashed box in $\mathrm{C}$. The serous exudate has leaked from the tumor $(\mathrm{T})$ through Bruch's membrane and the retinal pigment epithelium (arrowheads) and pushed the retina (R) away. Scale bar $1 \mathrm{~mm}$ 


\section{Methods}

\section{Patients and samples}

The study adhered to the tenets of the Declaration of Helsinki and the research group data security policy. Institutional Review Board approval was obtained from the Regional Ethical Review Board in Stockholm (Reference No. 2016/347-31/4, with amendment 2019-03485 approved by the Swedish Ethical Review Authority).

Seventy-five patients that had undergone enucleation between January 27th, 2000, and December 15th, 2007, were identified in our treatment registry along with retrospective data on gender, age at diagnosis, presenting symptoms, symptom duration before presentation, tumor thickness and tumor diameter, American Joint Committee on Cancer (AJCC) T-category, stage as well as dates of diagnosis, and last follow-up. At their first visit, patients had been asked about previous diseases, medications, family history, and symptoms. The attending ophthalmologist had generally not prompted the patient to confirm or deny a list of symptoms. Instead, the patient had been asked to describe his/her symptoms and their duration in own words. The ophthalmologist then recorded them as one or several of (1) blurred vision (decreased visual acuity), (2) shadow in the visual field, (3) photopsia and/or floaters, (4) metamorphopsia, (5) ocular pain, or (6) other, including photophobia, diplopia, and reduced perception of color. The tumor eye had also been examined with slit lamp biomicroscopy, ultrasonography, and fundus photography. The tumor's apical thickness, largest basal diameter (LBD), and location in the choroid had been noted. Primary enucleation is typically performed within 4 weeks after diagnosis.

Furthermore, tumor sections from these 75 patients were retrieved from the archives of the St. Erik Ocular Pathology Laboratory. For diagnostic purposes, these had previously been stained with hematoxylin and eosin, as well as PAS without hematoxylin counterstain. Sections from heavily pigmented tumors were bleached before staining. No new tissue was collected, sectioned, stained, or otherwise processed. After initial histological assessment, six patients were excluded ( $n=5$ no or too little tumor tissue represented in section; $n=1$ the represented tumor was fully necrotic). The tumor sections from the remaining 69 patients were then assessed for VM and scanned for digital measurement of PAS density according to a previously described method (Stålhammar et al. 2019).

\section{Follow-up}

After diagnosis, patients underwent radiological screening for metastases either with a combination of chest X-ray and an ultrasonography of the liver, or by computed tomography (CT) of the chest and abdomen. The metastasis screening of the liver was then repeated by ultrasonography or by CT semi-annually for a 5-year period after diagnosis. After enucleation, patients were scheduled for routine follow-up at 1,6 , and 12 months. If local control had been achieved, patients were then examined annually for the rest of their lives.

\section{Vasculogenic mimicry and PAS density}

Patterns of microvascular loops and networks were assessed in histological sections through a green narrow band-pass filter according to the method described by Folberg et al. (1992). The examining pathologist was blinded to all patient data, including symptoms and outcome. Presence of VM was defined as extracellular networks, closed loops, arcs with branching, or any combination of these. This definition replicates a distinction used in our previous publication, in which these patterns correlated strongly to PAS density, BAP-1 expression, gene expression class, and short metastasis-free survival (Stålhammar et al. 2019). Furthermore, the prognostic significance of the presence of loops, networks, and combined patterns have been verified in several publications from other laboratories (Foss et al. 1997a, b; McLean et al. 1997; Makitie et al. 1999).

PAS staining is mainly used for detection of structures containing a high proportion of carbohydrate macromolecules including glycogen, glycoproteins, and proteoglycans (Fu and Campbell-Thompson 2017). These are typically found in connective tissues, mucus, pericellular matrix, and basal laminae (Cerri and Sasso-Cerri 2003). Consequently, PAS will stain uveal melanoma blood vessels, but also a range of other tissue structures including vasculogenic mimicry, macrophages, and glycogen stored in the cytoplasm of tumor and non-tumor cells (Foss et al. 1997a, b; Nowak et al. 1998; Meersseman et al. 2011). The density of PAS-positive structures was evaluated with digital image analysis according to our previously described method (Stålhammar et al. 2019). In short, histological sections stained with PAS were scanned at $40 \times$, using a Nano Zoomer S60 (Hamamatsu Photonics K.K., Hamamatsu, Japan) at the Department of Pathology, Västmanland Hospital Västerås, Sweden. All tumor tissue in each section was then analyzed using QuPath Bioimage analysis v. 0.2.2 (Bankhead et al. 2017) for the density of blood vessels and extracellular matrix, defined as the number of PAS-positive pixels divided by the total number of pixels in the full tumor cross-section (Fig. 2). The sclera, Bruch's membrane, retina, vitreous and tumor areas 


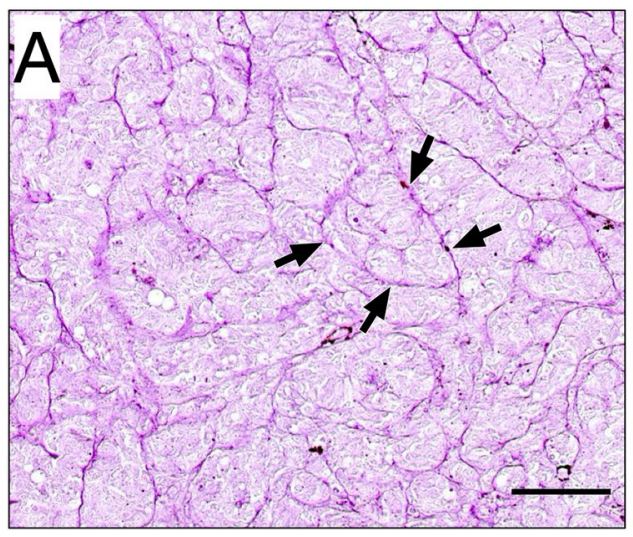

Fig. 2 Example of vasculogenic mimicry and digital image analysis of PAS density. Tissue section stained with Periodic acid-Schiff (PAS) without hematoxylin counterstain and digitally scanned at $\times 400$ (Nano Zoomer 2.0 HT, Hamamatsu Photonics K.K., Hamamatsu, Japan). A Patterns of vasculogenic mimicry can be seen,

with intense inflammation, abundant pigmentation, fibrosis, bleeding, necrosis, tissue folds, and areas of poor tissue fixation were excluded from analysis.

\section{Statistical methods}

When evaluated by the Shapiro-Wilk test, the deviation from normal distribution was not statistically significant for any of our continuous variables $(p>0.05)$ except PAS density $(p=0.001)$. We therefore used the Mann-Whitney $U$ test for the latter and independent samples Student's $t$ test when comparing all the other variables, with variances assumed according to Levene's test for equality of variances (equal variances assumed when $p>0.05$ ). The relationship between binary variables was examined with the Phi coefficient $(\varphi)$. For analysis of outcome in relation to presenting symptoms, the cumulative disease-specific survival was calculated. Disease-specific survival was defined as the proportion of patients not deceased from uveal melanoma. Median followup was defined as the median time (years) from diagnosis to the date of data collection for patients that were alive according to the national population register. Differences with a $p<0.05$ were considered significant, all $p$ values being twosided. All statistical analyses were performed using SPSS statistics version 27 (IBM, Armonk, NY, USA).

\section{Results}

\section{Descriptive statistics}

A total of 69 patients with 69 tumors were included. Their mean age at diagnosis was 63 years (standard deviation, SD 12), and the mean tumor thickness and diameter $8.2 \mathrm{~mm}$ (SD

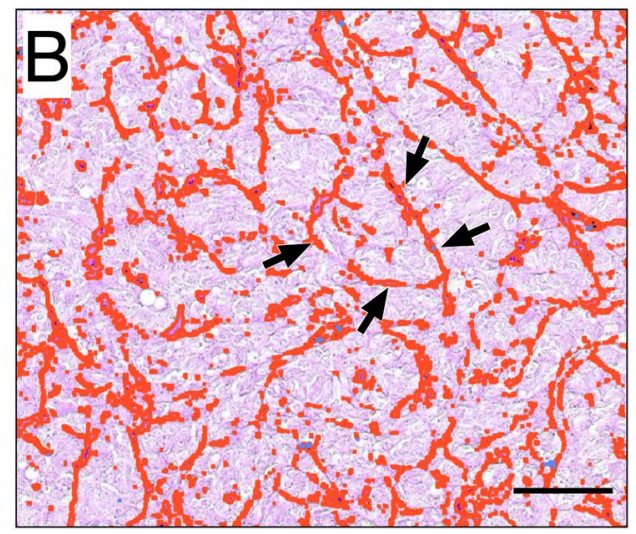

including arches (arrows). Assessments of the presence of vasculogenic mimicry were made in a light microscope with a green narrow band-pass filter (not shown in this digitally scanned section). B With digital image analysis of the same tumor area, the patterns have been automatically detected, highlighted and quantified. Scale bars $0.1 \mathrm{~mm}$

3.2) and $14.3 \mathrm{~mm}$ (SD 4.4), respectively. Most tumors (43 of $69,62 \%$ ) were of American Joint Committee on Cancer (AJCC) T-category 2 or 3 . The median time elapsed from diagnosis to enucleation was 0.4 months (mean 4.6, SD 7.5). Twenty-three patients (33\%) had undergone plaque brachytherapy prior to enucleation. The reason for the secondary enucleation was tumor progression or lack of regression after brachytherapy in 15 , intraocular bleeding and/or elevated intraocular pressure in four, very low visual function in two, pain in one, and a scleral abscess in one. Fifty-three of 69 patients (77\%) had deceased before the end of followup, of which 36 (52\% of full cohort, $68 \%$ of deaths) died of metastasized uveal melanoma. Median follow-up for the 13 survivors was 16.7 years (SD 2.6, Table 1).

Thirty four of 69 patients (49\%) presented with blurred vision. $18(26 \%)$ with a shadow in the visual field, $7(10 \%)$ with photopsia and/or floaters, 2 (3\%) with metamorphopsia, and 0 presented with ocular pain and/or with other symptoms. Nine patients (13\%) had no symptoms at all (Table 2). The mean symptom duration before diagnosis was 4 months (SD 5).

\section{Vasculogenic mimicry}

Patterns of VM were identified in 23 of 69 tumors (33\%). VM was identified in 6 of 34 patients $(18 \%)$ that presented with blurred vision; in 16 of 18 patients $(89 \%)$ with a shadow in the visual field; in 0 of 7 patients with photopsia and/or floaters; in 0 of 2 patients with metamorphopsia; and in 1 of 9 patients (11\%) that were asymptomatic (Fig. 3). Tumors with VM did not have larger diameters than tumors without VM (15.0 and $12.8 \mathrm{~mm}$, respectively, Student's $t$ test $\mathrm{p}=0.09)$. Similarly, tumors with VM did not have greater 
Table 1 Demographics and clinical features of study patients and tumors

\begin{tabular}{ll}
\hline$n=$ & 69 \\
Mean age at diagnosis, years (SD) & $63(12)$ \\
Sex, $n$ (\%) & \\
Female & $27(39)$ \\
Male & $43(61)$ \\
Tumor eye laterality, $n$ (\%) & \\
Right & $35(51)$ \\
Left & $34(49)$ \\
Months from diagnosis to enucleation, median (SD) & $0.4(7.5)$ \\
Previous brachytherapy, $n$ (\%) & $23(33)$ \\
Mean tumor thickness, mm (SD) & $8.2(3.2)$ \\
Mean tumor diameter, mm (SD) & $14.3(4.4)$ \\
Mean tumor distance to optic disc, mm (SD) & $3.2(3.8)$ \\
AJCC T-category, $n(\%)$ & \\
1 & $9(13)$ \\
2 & $20(29)$ \\
3 & $23(33)$ \\
4 & $17(25)$ \\
AJCC stage, $n(\%)$ & \\
I & $9(13)$ \\
IIA & $20(29)$ \\
IIB & $23(33)$ \\
IIIA & $17(25)$ \\
IIIB & $0(0)$ \\
IV & $0(0)$ \\
Median follow-up, years (SD, min.-max.) & $0(0)$ \\
& 16.7 y $(2.6$, \\
& $12.5-$ \\
& $20.3)$ \\
\hline
\end{tabular}

$S D$ standard deviation, AJCC American Joint Committee on Cancer

Table 2 Distribution of presenting symptoms

\begin{tabular}{ll}
\hline Symptom & $n(\%)$ \\
\hline Blurred vision & $34(49)$ \\
Shadow in visual field & $18(26)$ \\
Photopsia and/or floaters & $7(10)$ \\
Metamorphopsia & $2(3)$ \\
Ocular pain & $0(0)$ \\
Other & $0(0)$ \\
No symptoms & $9(13)$ \\
\hline
\end{tabular}

apical thickness than tumors without VM $(8.3$ and $7.8 \mathrm{~mm}$, respectively, $p=0.56$ ).

The presence of VM correlated to a shadow as a presenting symptom ( $\varphi 0.70, p<0.001$, Table $3 a)$ but not with ocular pain, metamorphopsia, or other symptoms $(p>0.31)$. Blurred vision and photopsia and/or floaters were negatively correlated $(\varphi-0.33, p=0.006$ and $\varphi-0.24, p=0.048$, respectively).

\section{PAS density}

The mean PAS density in all 69 tumors was 6.1\% (SD 6.3, $\min 0.4 \%$, $\max 37.9 \%$ ). Patients presenting with a shadow in the visual field had greater PAS density than patients without a shadow (11.1 versus 4.5\%, Mann-Whitney $U p<0.001$, Fig. 4A).

On the other hand, patients presenting with blurred vision had lower PAS density than patients without blurred vision ( $4.4 \%$ versus $7.8 \%, p=0.03$, Fig. 4B). Similarly, patients presenting with metamorphopsia had lower PAS density than patients without metamorphopsia $(0.5 \%$ versus $6.3 \%$, $p=0.002$, Fig. 4C).

Patients presenting with photopsia and/or floaters had similar PAS density to patients without photopsia or floaters $(4.3 \%$ versus $6.3 \%, p=0.41$, Fig. $4 \mathrm{D})$.

PAS density $\geq$ median correlated to a shadow as a presenting symptom $(\varphi 0.50, p<0.001$, Table $3 b)$ but not with blurred vision, ocular pain, metamorphopsia, photopsia and/ or floaters, or other symptoms ( $p=0.40-0.99)$. PAS density $\geq$ median also correlated to presence of VM ( $\varphi 0.55$, $p<0.001$ ). Tumors with PAS density $\geq$ median did not have larger diameters than tumors with PAS density $<$ median (13.4 and $15.3 \mathrm{~mm}$, respectively, $p=0.14$ ). Similarly, tumors with PAS density $\geq$ median did not have greater apical thickness than tumors with PAS density $<$ median $(8.4$ and $7.9 \mathrm{~mm}$, respectively, $p=0.58$ ).

\section{Shadow versus tumor histology}

Patients presenting with a shadow in the visual field had tumors with similar apical thickness, diameter, distance to the optic disc, distance to the macula, AJCC T-category, and proportion of ciliary body engagement as patients that did not experience a shadow. However, the former had a significantly higher proportion of retinal detachments, and of tumors displaying vasculogenic mimicry and PAS density $\geq$ median (Table 4).

In a multivariate regression with all of tumor distance to the macula, tumor apical thickness, tumor diameter, retinal detachment, ciliary body engagement, presence of vasculogenic mimicry and PAS density entered as covariates, vasculogenic mimicry, and PAS density $\geq$ median, but none of the others were independent predictors of a shadow in the visual field. Ciliary body engagement was a negative predictor (Table 5). 
Fig. 3 Examples of tumors with and without vasculogenic mimicry. $\mathbf{A}$ and $\mathbf{B}$ In these two tumors, no vasculogenic mimicry was identified. Tumor surface is relatively homogenous with PAS-positive patterned extracellular matrix. $\mathbf{C}$ and $\mathbf{D}$ In two other tumors, vasculogenic mimicry was identified, defined as extracellular networks, closed loops, arcs with branching, or any combination of these. Some of these patterns are indicated (arrows). Assessments of the presence of vasculogenic mimicry were made in a light microscope with a green narrow band-pass filter (not shown in this digitally scanned section). Scale bars $0.25 \mathrm{~mm}$
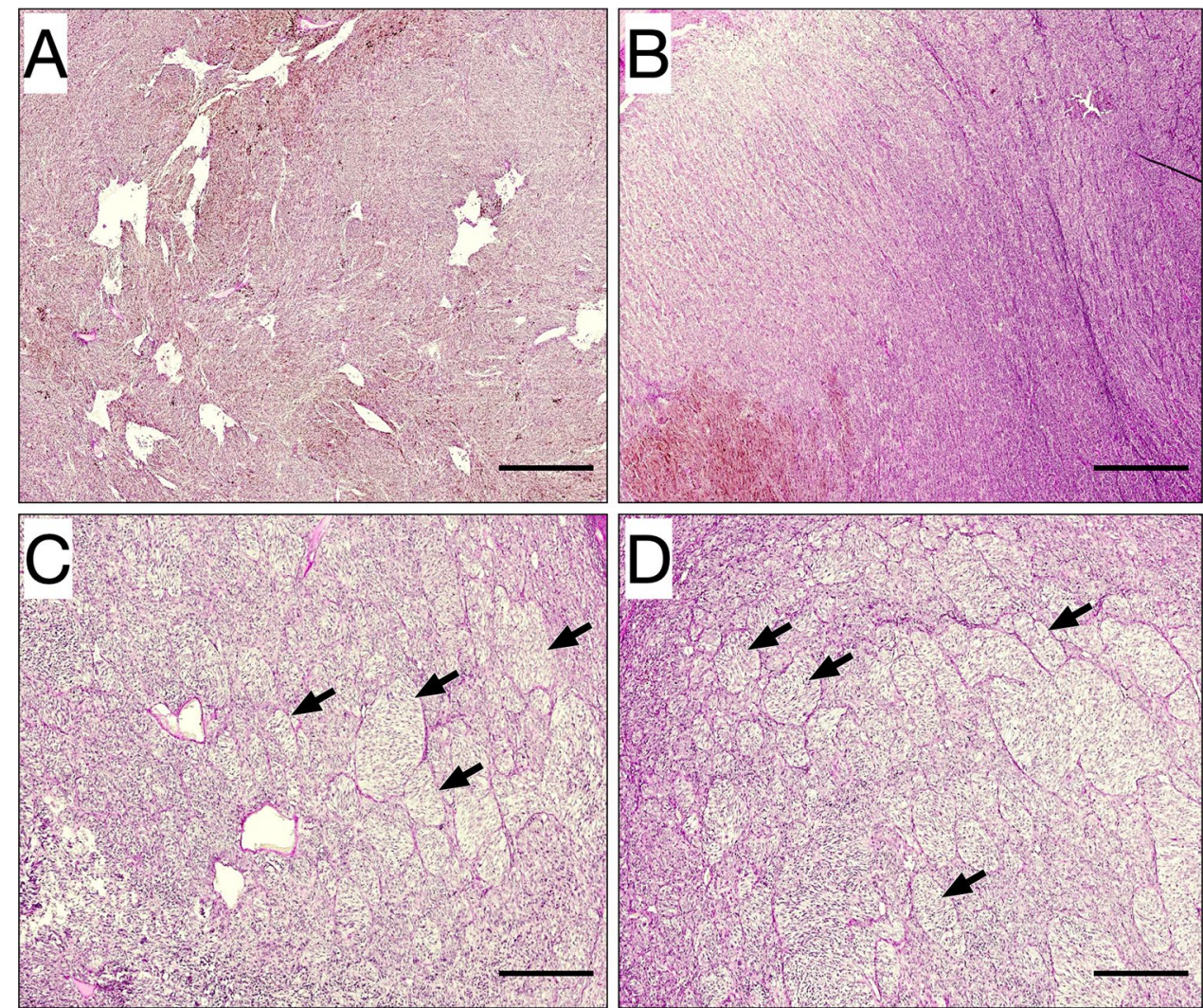

Table 3 Presence of vasculogenic mimicry and PAS density in relation to a shadow in the visual field as presenting symptom.

a. Shadow in the visual field vs. vasculogenic mimicry

\begin{tabular}{llll}
\hline & Vasculogenic mimicry present & Vasculogenic mimicry not present & Total \\
\hline Shadow in the visual field & 16 & 1 & 17 \\
No shadow in the visual field & 5 & 47 & 52 \\
Total & 21 & 48 & 69 \\
\hline
\end{tabular}

b.Shadow in the visual field vs. PAS density

\begin{tabular}{llll}
\hline & PAS density $\geq$ median & PAS density $<$ median & Total \\
Shadow in the visual field & 16 & 1 & 17 \\
No shadow in the visual field & 19 & 33 & 52 \\
Total & 35 & 34 & 69 \\
\hline
\end{tabular}

\section{Survival}

No presenting symptom $(p=0.54-0.75)$ or $\mathrm{RD}(p=0.28)$ was associated with shortened disease-specific survival in the present cohort. The presence of VM was associated with significantly shorter disease-specific survival $(p=0.04$, Fig. 5a), but not PAS density $\geq$ median ( $p=0.65$, Fig. $5 b)$.

\section{Discussion}

In this study, a shadow in the visual field as a presenting symptom of uveal melanoma, experienced by one in four patients, correlated strongly with patterns of VM and increased PAS density. Both of which are markers of 

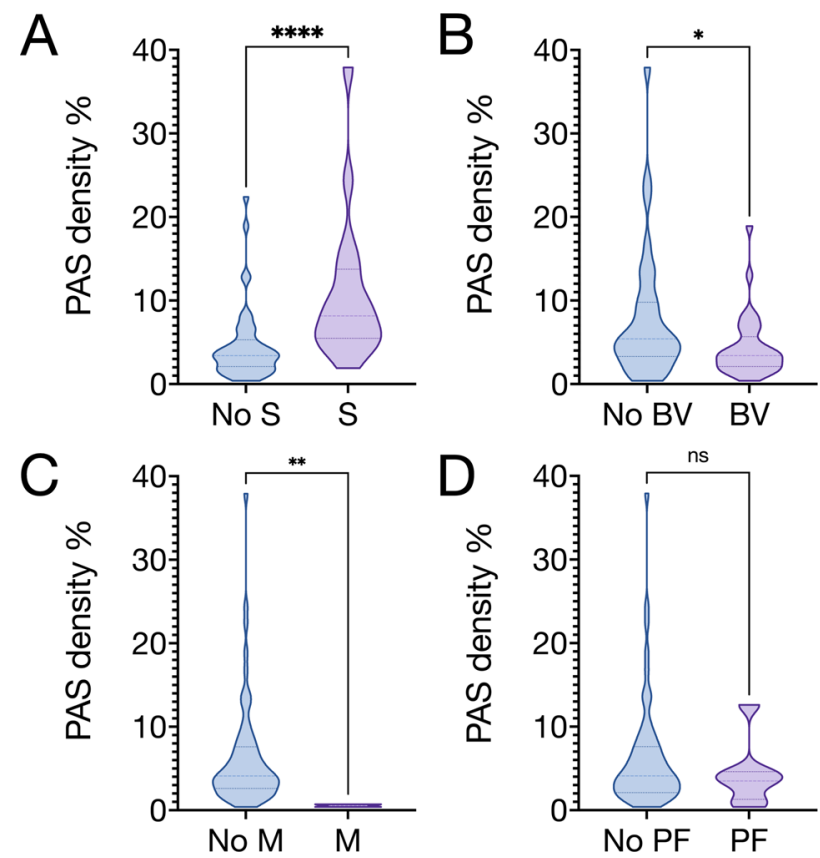

Fig. 4 Violin plots, PAS density in relation to presenting symptoms of uveal melanoma. PAS density was defined as the number of PASpositive pixels divided by the total number of pixels in the full tumor cross-section, as measured with digital image analysis. A Patients presenting with a shadow in the visual field (S) had greater PAS density than patients without a shadow (11.1 versus $4.5 \%$, Mann-Whitney $U p<0.0001)$. B Patients presenting with blurred vision (BV) had lower PAS density than patients without blurred vision (4.4\% versus $7.8 \%, p=0.03)$. C Patients presenting with metamorphopsia (M) had lower PAS density than patients without metamorphopsia $(0.5 \%$ versus $6.3 \%, p=0.002)$. D Patients presenting with photopsia and/or floaters (PF) had similar PAS density to patients without photopsia or floaters $(4.3 \%$ versus $6.3 \%, p=0.41)$. $n s$ non-significant on a 0.05 level. ${ }^{*} p<0.05$. ${ }^{* *} p<0.01$. ${ }^{* * *} p<0.001$. ${ }^{* * * *} p<0.0001$

aggressive disease (Foss et al. 1997a, b; Mäkitie et al. 1999; Kivelä et al. 2004; Stålhammar et al. 2019).

In 1999, Mäkitie et al. found a significant association between VM, microvascular density and exudative retinal detachment, independent of tumor size and ciliary body involvement (Mäkitie et al. 1999). Furthermore, when adjusting for the latter two factors, retinal detachment did not predict poor patient survival (Kivela et al. 2001). Consequently, we believe that there is no coincidence that we found a strong correlation between a shadow in the visual field, VM, and PAS density, and that in turn, this correlation may be the causal link between the shadow and poor prognosis. As we have shown previously, VM also correlates to gene expression class, expression of BAP-1, and macrophage infiltration (See et al. 2019; Stålhammar et al. 2019).

Gene expression profiles are of great importance in stratifying patient prognosis. Furthermore, mutations in the tumor suppressor BRCA1-associated protein-1 gene (BAP1), located on chromosome $3 \mathrm{p}$, are mutated in a vast majority of metastasizing UM (Harbour et al. 2010; Karlsson et al. 2020). This mutation leads to a dedifferentiated stem-like phenotype that correlates strongly with poor prognosis (Harbour et al. 2010; Karlsson et al. 2020). BAPl is one of several genes that is associated with epithelial-to-mesenchymal transition (EMT) of the tumor cells. In this mutation sequence, the BAPl mutation has been assumed to occur relatively late, preceded by mutations in G-protein subunits including GNAII and GNAQ that are present in as high as 83-96\% of UM (Decatur et al. 2016). Recently, however, we estimated that the BAP1 mutation occurs when the primary tumor has a size of only a few malignant cells to $6 \mathrm{~mm}^{3}$ (Uner et al. 2021). Furthermore, other genetic aberrations may underlie the VM phenotype and increased PAS density. The role of non-coding RNAs has gained attention, including dysregulation of the possible oncogene LINC00518 which may contribute to EMT, hypoxia-induced responses and thereby potentially vascularity and leakiness (Barbagallo et al. 2020).

PAS density should not be immediately translated into a representative of microvascular density, as a multitude of structures other than endothelial-lined blood vessels may be
Table 4 Tumor characteristics in relation to visual field shadow

\begin{tabular}{lllc}
\hline & Shadow $(n=18)$ & No shadow $(n=51)$ & $p^{*}$ \\
\hline Mean tumor apical thickness, mm (SD) & $8.1(3.5)$ & $7.9(3.2)$ & 0.86 \\
Mean tumor diameter, mm (SD) & $14.5(4.7)$ & $13.6(4.4)$ & 0.47 \\
Mean tumor distance to optic disc, mm (SD) & $4.3(4.2)$ & $4.5(5.1)$ & 0.90 \\
Mean tumor distance to macula, mm (SD) & $5.6(4.7)$ & $4.4(4.3)$ & 0.34 \\
Mean AJCC T-category 1-4 (SD) & $2.4(0.9)$ & $2.8(1.0)$ & 0.18 \\
Ciliary body engagement, $n(\%)$ & $3(17)$ & $11(22)$ & 0.75 \\
Retinal detachment, $n(\%)$ & $14(78)$ & $21(41)$ & 0.013 \\
Vasculogenic mimicry present, $n(\%)$ & $16(89)$ & $7(14)$ & $<0.0001$ \\
PAS density $\geq$ median, $n(\%)$ & $17(94)$ & $19(37)$ & $<0.0001$ \\
\hline
\end{tabular}

$S D$ standard deviation

*By Fisher's exact test for categorical variables and Student's T test for continuous variables 
Table 5 Multivariate regression, hazard for shadow as presenting symptom

\begin{tabular}{lrcccccc}
\hline Covariate & $B$ & S.E & Wald & $p$ & Exp(B) & 95\% CI lower & 95\% CI upper \\
\hline Distance from macula, mm & 0.3 & 0.2 & 2.6 & 0.11 & 1.3 & 0.9 & 1.8 \\
Tumor diameter, mm & 0.05 & 0.1 & 0.1 & 0.74 & 1.1 & 0.8 & 1.4 \\
Tumor thickness, mm & -0.2 & 0.2 & 0.4 & 0.53 & 0.9 & 0.5 & 1.4 \\
Retinal detachment & 2.5 & 1.4 & 3.1 & 0.08 & 12.3 & 0.7 & 206.1 \\
Ciliary body engagement & -4.9 & 2.2 & 5.1 & 0.02 & 0.007 & 0.0 & 0.5 \\
Vasculogenic mimicry present & 4.8 & 1.5 & 11.1 & 0.001 & 124.9 & 7.3 & 2149.6 \\
PAS density & 3.2 & 1.5 & 4.4 & 0.04 & 23.7 & 1.2 & 462.7 \\
Constant & -6.8 & 2.8 & 6.0 & 0.01 & 0.001 & &
\end{tabular}

positively stained with PAS, including vasculogenic mimicry, macrophages, and glycogen stored in the cytoplasm of tumor and non-tumor cells (Foss et al. 1997a, b; Nowak et al. 1998; Meersseman et al. 2011). It is therefore important that the lack of prognostic significance of PAS density is not used to cast doubt on the prognostic significance of microvascular density.

The other presenting symptoms investigated by us are not associated with tumor vascularity or with patient outcome, neither in cumulative survival analysis nor in multivariate Cox regressions (Fili et al. 2021). Notably, blurred vision has been the most common symptom in our studies, with similar prevalence reported in a previous publication by Damato and Coupland (2012). Metamorphopsia, ocular pain and other symptoms were exceptions, each experienced by only 1 or $2 \%$ of patients.

There are several limitations to this study. The patients were included from one institution only and the data included were retrospective in nature, which limits our control over confounding factors. There is a range of other important prognostic factors in uveal melanoma that were not available for comparison in this study. Among these, analyses of the immunohistochemical expression of BAP-1 and P16INK4a, mutations in the BAPl gene, and gene expression classifications in relation to VM, PAS density, and presenting symptoms would have been highly interesting (Harbour et al. 2010; Onken et al. 2012; Russo et al. 2020; Herrspiegel et al. 2021). Our cohort was considerably smaller than our previous cohort in which the association between symptom and prognosis was found, which is the probable reason for the lack of survival differences between patients with and without specific symptoms herein, and for the non-significant difference in tumor diameters between tumors with and without VM. One-third of the included tumors had been previously treated with plaque brachytherapy, which may alter histological appearance. A previous case-control study has found a lower microvascular density in irradiated versus nonirradiated uveal melanomas (Toivonen et al. 2003). However, it cannot be excluded that this signifies a difference at baseline. We have no data on the growth rate of the tumors included, which may have offered alternative explanations to presenting symptoms and lack thereof. Furthermore, the description of a symptom is by definition based on a subjective experience and was not confirmed by additional testing, including perimetry. Some patients that reported a shadow may have experienced what others would describe as a floater or other, etc. Finally, even though any number of symptoms could be recorded by the patients in this study, it is possible that any symptom either is not noticed or not reported if it is obscured by other simultaneous symptoms. In that case, the true prevalence of symptoms would be higher than what we have reported here.

In conclusion, blurred vision is the most common presenting symptom in uveal melanoma. Metamorphopsia, pain, and other symptoms are all relatively rare. Patients presenting with a shadow have tumors with greater PAS density and are highly likely to have VM, likely explaining the previously reported association between this symptom and poor prognosis. 
Fig. 5 Cumulative diseasespecific survival proportions. A Patients with presence of VM in their tumors (red) had significantly shorter disease-specific survival than patients without VM (green, Wilcoxon $p=0.04$ ). B PAS density was not associated with disease-specific survival $(\mathbf{p}=0.65)$. Colored areas represent $95 \%$ confidence intervals. $V M$ vasculogenic mimicry

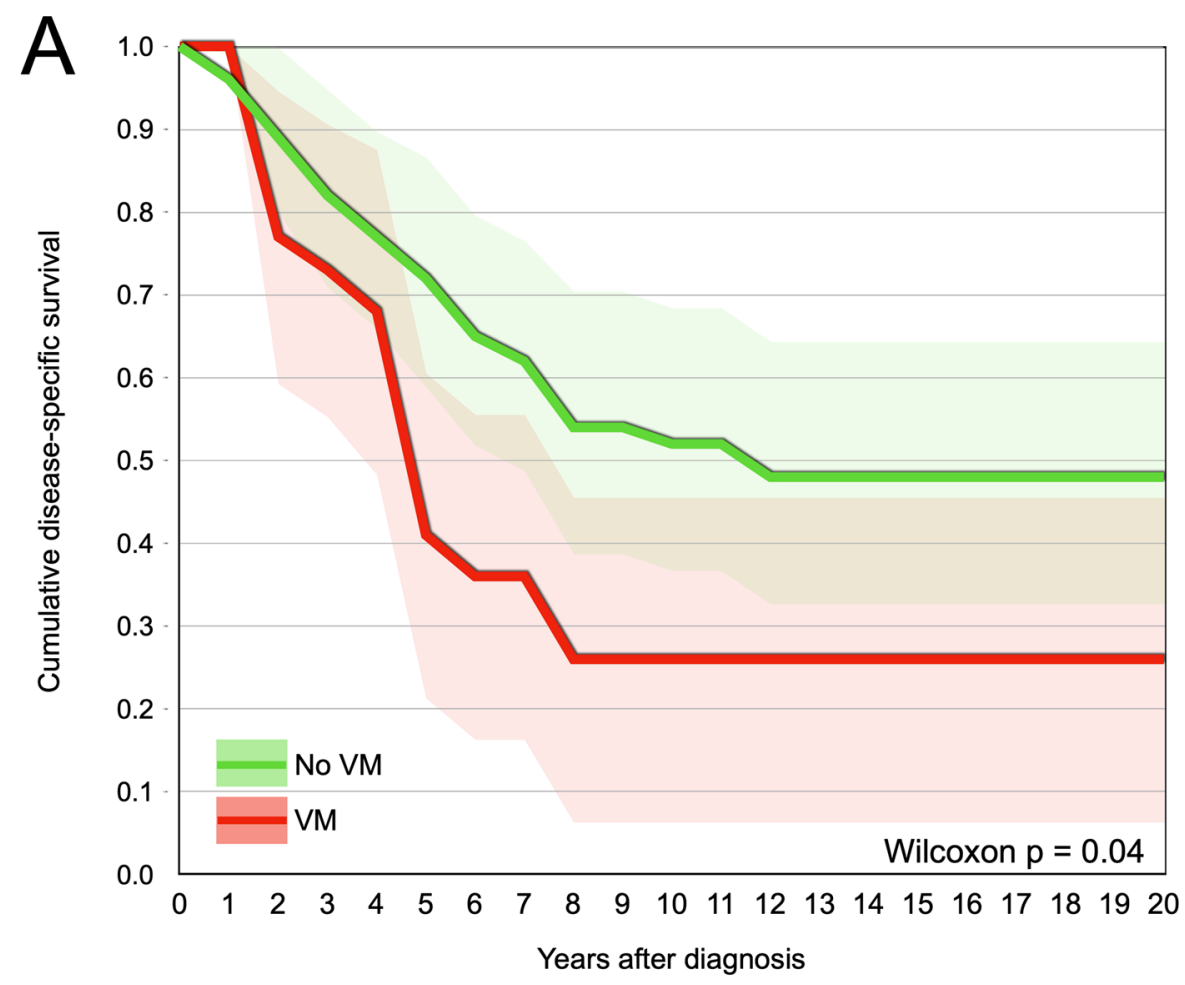

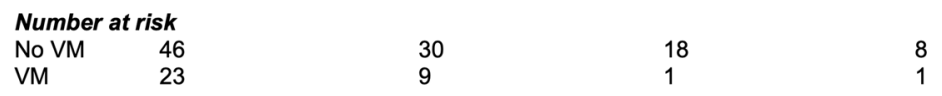

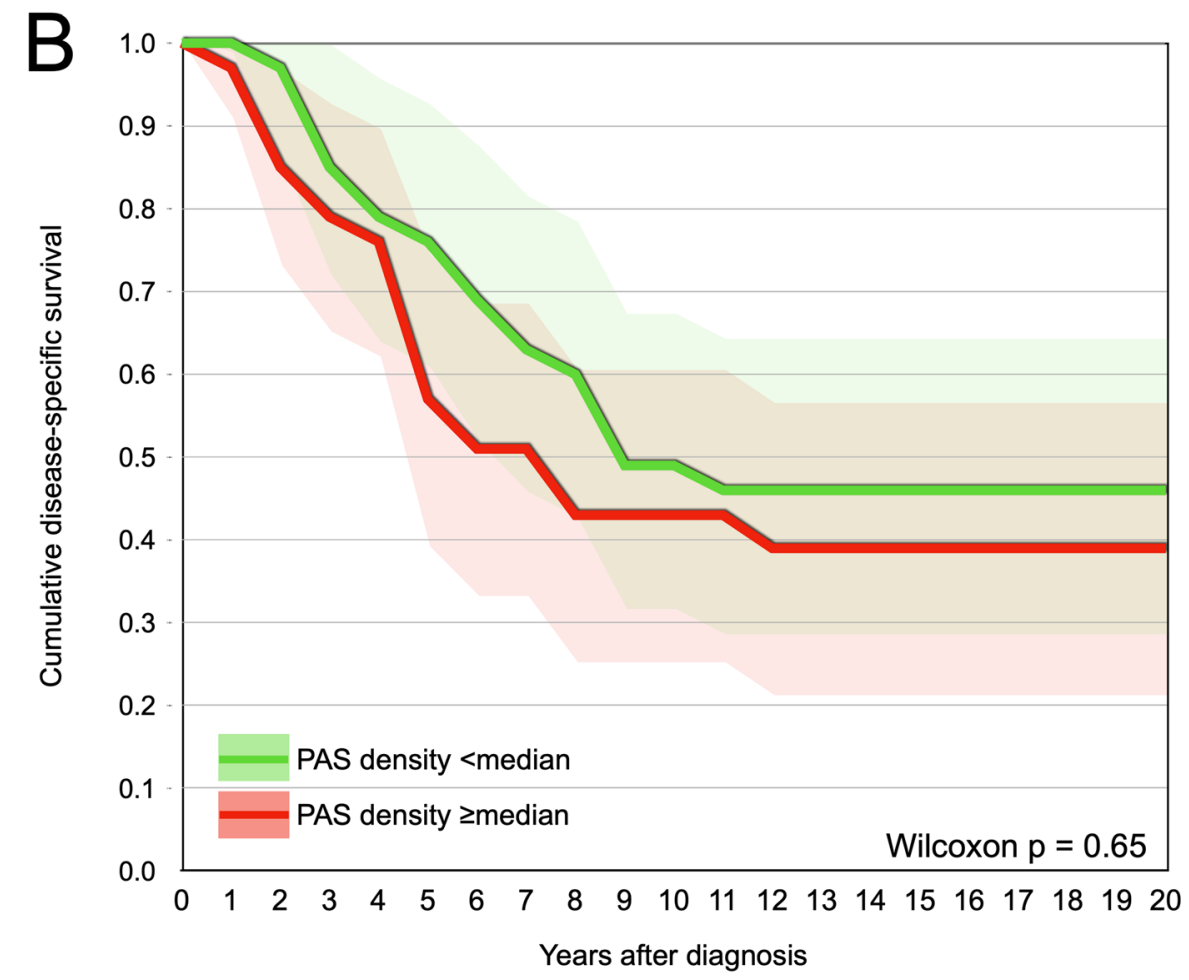

Number at risk

$\geq$ median $\quad 36$

18

11
12

2
7 
Author contributions SS, $\mathrm{CH}$ and GS collected patient data. VG scanned the tissue sections. GS performed the histological examinations and digital image analysis. All authors contributed to the writing of the manuscript, read, and approved the final version.

Funding Open access funding provided by Karolinska Institute. Support for this study was provided to Gustav Stålhammar from: the Royal Swedish Academy of Sciences (reference ME2019-0036), The Swedish Cancer Society (20 0798 Fk), The Swedish Society of Medicine (Cronqvists stiftelse, Reference SLS 934014), The Swedish Eye Foundation (Reference 2020-04-27), Karolinska Institutet (Reference 201901900, 2020-013333, and 2020-02517), Region Stockholm (Reference 20200356), The Crown Princess Margareta Foundation for the Visually Impaired (Reference 157), and Carmen and Bertil Regnér Foundation (Reference 2020-00062). The funding organizations had no role in the design or conduct of this research.

Availability of data and materials The datasets used and/or analyzed during the current study are available from the corresponding author on reasonable request.

\section{Declarations}

Conflict of interest The authors declare that they have no competing interests.

Ethics approval and consent to participate Institutional Review Board approval was obtained from the regional ethical review board in Stockholm (Reference No. 2016/347-31/4, with amendment 2019-03485 approved by the Swedish Ethical Review Authority). Informed consent was waived as this is a retrospective chart review that did not affect the treatment or follow-up of patients and did not require new collection of sensitive data. Furthermore, no new tissues were collected, sectioned, stained, or otherwise processed.

\section{Consent for publication Not applicable.}

Open Access This article is licensed under a Creative Commons Attribution 4.0 International License, which permits use, sharing, adaptation, distribution and reproduction in any medium or format, as long as you give appropriate credit to the original author(s) and the source, provide a link to the Creative Commons licence, and indicate if changes were made. The images or other third party material in this article are included in the article's Creative Commons licence, unless indicated otherwise in a credit line to the material. If material is not included in the article's Creative Commons licence and your intended use is not permitted by statutory regulation or exceeds the permitted use, you will need to obtain permission directly from the copyright holder. To view a copy of this licence, visit http://creativecommons.org/licenses/by/4.0/.

\section{References}

Asnaghi L, Lin MH, Lim KS, Lim KJ, Bar EE, Eberhart CG (2013) Targeting the hypoxia pathway in uveal melanoma cells. Acta Ophthalmol 91:0

Bankhead P, Loughrey M, Fernández J, Dombrowski Y, McArt D, Dunne P, McQuaid S, Gray R, Murray L, Coleman H, James J, Salto-Tellez M, Hamilton P (2017) QuPath: open source software for digital pathology image analysis. Sci Rep 7:16878-16878

Barbagallo C, Caltabiano R, Broggi G, Russo A, Puzzo L, Avitabile T, Longo A, Reibaldi M, Barbagallo D, Di Pietro C, Purrello M, Ragusa M (2020) LncRNA LINC00518 acts as an oncogene in uveal melanoma by regulating an RNA-based network. Cancers 12:3867

Callejo SA, Antecka E, Blanco PL, Edelstein C, Burnier MN Jr (2007) Identification of circulating malignant cells and its correlation with prognostic factors and treatment in uveal melanoma. A prospective longitudinal study. Eye 21:752-759

Cao Z, Bao M, Miele L, Sarkar FH, Wang Z, Zhou Q (2013) Tumour vasculogenic mimicry is associated with poor prognosis of human cancer patients: a systemic review and meta-analysis. Eur J Cancer 49:3914-3923

Cerri PS, Sasso-Cerri E (2003) Staining methods applied to glycol methacrylate embedded tissue sections. Micron 34:365-372

COMS (2006) The COMS randomized trial of iodine 125 brachytherapy for choroidal melanoma: V. twelve-year mortality rates and prognostic factors: COMS report no. 28. Arch Ophthalmol 124:1684-1693

Damato BE, Coupland SE (2012) Differences in uveal melanomas between men and women from the British Isles. Eye 26:292-299

Damato EM, Damato BE (2012) Detection and time to treatment of uveal melanoma in the United Kingdom: an evaluation of 2,384 patients. Ophthalmology 119:1582-1589

Decatur CL, Ong E, Garg N, Anbunathan H, Bowcock AM, Field MG, Harbour JW (2016) Driver mutations in uveal melanoma: associations with gene expression profile and patient outcomes. JAMA Ophthalmol 134:728-733

Fili M, Seregard S, Stalhammar G (2021) Presenting symptoms are associated with uveal melanoma-related death. Ophthalmology 128:1107-1109. https://doi.org/10.1016/j.ophtha.2020.11.023

Folberg R, Maniotis AJ (2004) Vasculogenic mimicry. APMIS 112:508-525

Folberg R, Pe'Er J, Gruman LM, Woolson RF, Jeng G, Montague $\mathrm{PR}$, Moninger TO, Yi H, Moore KC, Montague PR, Moninger TO, Yi H, Moore KC (1992) The morphologic characteristics of tumor blood vessels as a marker of tumor progression in primary human uveal melanoma: a matched case-control study. Hum Pathol 23:1298-1305

Folberg R, Fleck M, Mehaffey MG, Meyer M, Bentler SE, Woolson RF, Pe'er J (1996) Mapping the location of prognostically significant microcirculatory patterns in ciliary body and choroidal melanomas. Pathol Oncol Res 2:229-236

Foss AJ, Alexander RA, Jefferies LW, Hungerford JL, Harris AL, Lightman S (1996) Microvessel count predicts survival in uveal melanoma. Can Res 56:2900

Foss AJ, Whelehan I, Hungerford JL, Anderson DF, Errington RD, Kacperek A, Restori M, Kongerud J, Sheen M (1997a) Predictive factors for the development of rubeosis following proton beam radiotherapy for uveal melanoma. Br J Ophthalmol 81:748-754

Foss AJE, Alexander RA, Hungerford JL, Harris AL, Cree IA, Lightman S (1997b) Reassessment of the PAS patterns in uveal melanoma. Br J Ophthalmol 81:240

Fu DA, Campbell-Thompson M (2017) Periodic acid-Schiff staining with diastase. Methods Mol Biol 1639:145-149

Garg G, Finger PT, Kivela TT, Simpson ER, Gallie BL, Saakyan S, Amiryan AG, Valskiy V, Chin KJ, Semenova E, Seregard S, Fili M, Wilson M, Haik B, Caminal JM, Catala-Mora J, Gutierrez C, Pelayes DE, Folgar AM, Jager MJ, Dogrusoz M, Luyten GPM, Singh AD, Suzuki S, AOOT Force (2021) Patients presenting with metastases: stage IV uveal melanoma, an international study. Br J Ophthalmol. https://doi.org/10.1136/bjophthalmol-2020-317949

Harbour JW, Onken MD, Roberson ED, Duan S, Cao L, Worley LA, Council ML, Matatall KA, Helms C, Bowcock AM (2010) Frequent mutation of BAP1 in metastasizing uveal melanomas. Science 330:1410-1413

Herrspiegel C, Kvanta A, Lardner E, Ramskold Cabaca L, Wells J, Bartuma K, Seregard S, Stalhammar G (2021) Nuclear expression of BAP-1 in transvitreal incisional biopsies and subsequent 
enucleation of eyes with posterior choroidal melanoma. Br J Ophthalmol 105:582-586

Karlsson J, Nilsson LM, Mitra S, Alsen S, Shelke GV, Sah VR, Forsberg EMV, Stierner U, All-Eriksson C, Einarsdottir B, Jespersen H, Ny L, Lindner P, Larsson E, Olofsson Bagge R, Nilsson JA (2020) Molecular profiling of driver events in metastatic uveal melanoma. Nat Commun 11:1894

Khoja L, Atenafu EG, Suciu S, Leyvraz S, Sato T, Marshall E, Keilholz U, Zimmer L, Patel SP, Piperno-Neumann S, Piulats J, Kivela TT, Pfoehler C, Bhatia S, Huppert P, Van Iersel LBJ, De Vries IJM, Penel N, Vogl T, Cheng T, Fiorentini G, Mouriaux F, Tarhini A, Patel PM, Carvajal R, Joshua AM (2019) Meta-analysis in metastatic uveal melanoma to determine progression free and overall survival benchmarks: an international rare cancers initiative (IRCI) ocular melanoma study. Ann Oncol 30:1370-1380

Kivela T, Eskelin S, Makitie T, Summanen P (2001) Exudative retinal detachment from malignant uveal melanoma: predictors and prognostic significance. Invest Ophthalmol vis Sci 42:2085-2093

Kivelä T, Mäkitie T, Rana'a T, Toivonen P (2004) Microvascular loops and networks in uveal melanoma. Can J Ophthalmol 39:409-421

Kujala E, Mäkitie T, Kivelä T (2003) Very long-term prognosis of patients with malignant uveal melanoma. Invest Ophthalmol vis Sci 44:4651-4659

Mäkitie T, Summanen P, Tarkkanen A, Kivelä T (1999) Microvascular density in predicting survival of patients with choroidal and ciliary body melanoma. Invest Ophthalmol vis Sci 40:2471

Makitie T, Summanen P, Tarkkanen A, Kivela T (1999) Microvascular loops and networks as prognostic indicators in choroidal and ciliary body melanomas. J Natl Cancer Inst 91:359-367

McLean IW, Keefe KS, Burnier MN (1997) Uveal melanoma. Comparison of the prognostic value of fibrovascular loops, mean of the ten largest nucleoli, cell type, and tumor size. Ophthalmology 104:777-780

Meersseman W, Verschueren P, Tousseyn T, De Vos R, Cassiman D (2011) PAS-positive macrophages-not always infection. Lancet 377:1890

Nowak MA, Fatteh SM, Campbell TE (1998) Glycogen-rich malignant melanomas and glycogen-rich balloon cell malignant melanomas: frequency and pattern of PAS positivity in primary and metastatic melanomas. Arch Pathol Lab Med 122:353-360
Onken MD, Worley LA, Char DH, Augsburger JJ, Correa ZM, Nudleman E, Aaberg TM, Altaweel MM, Bardenstein DS, Finger PT, Gallie BL, Harocopos GJ, Hovland PG, McGowan HD, Milman T, Mruthyunjaya P, Simpson ER, Smith ME, Wilson DJ, Wirostko WJ, Harbour JW (2012) Collaborative Ocular Oncology Group report number 1: prospective validation of a multi-gene prognostic assay in uveal melanoma. Ophthalmology 119:1596-1603

Packer S, Stoller S, Lesser ML, Mandel FS, Finger PT (1992) Longterm results of iodine 125 irradiation of uveal melanoma. Ophthalmology 99:767-773 (discussion 774)

Rantala ES, Hernberg M, Kivela TT (2019) Overall survival after treatment for metastatic uveal melanoma: a systematic review and meta-analysis. Melanoma Res 29:561-568

Russo D, Di Crescenzo RM, Broggi G, Merolla F, Martino F, Varricchio S, Ilardi G, Borzillo A, Carandente R, Pignatiello S, Mascolo M, Caltabiano R, Staibano S (2020) Expression of P16INK4a in uveal melanoma: new perspectives. Front Oncol 10:562074

See TRO, Stålhammar G, Phillips SS, Grossniklaus HE (2019) BAP1 immunoreactivity correlates with gene expression class in uveal melanoma. Ocul Oncol Pathol 6:1-9

Singh AD (2001) Uveal melanoma: implications of tumor doubling time. Ophthalmology 108:829-830

Stålhammar G, See TRO, Phillips SS, Grossniklaus HE (2019) Density of PAS positive patterns in uveal melanoma: Correlation with vasculogenic mimicry, gene expression class, BAP-1 expression, macrophage infiltration, and risk for metastasis. Mol vis 25:502-516

Stalhammar G (2020) Forty-year prognosis after plaque brachytherapy of uveal melanoma. Sci Rep 10:11297

Toivonen P, Makitie T, Kujala E, Kivela T (2003) Macrophages and microcirculation in regressed and partially regressed irradiated choroidal and ciliary body melanomas. Curr Eye Res 27:237-245

Uner OE, See TRO, Szalai E, Grossniklaus HE, Stalhammar G (2021) Estimation of the timing of BAP1 mutation in uveal melanoma progression. Sci Rep 11:8923

Publisher's Note Springer Nature remains neutral with regard to jurisdictional claims in published maps and institutional affiliations. 